\title{
REVIEW
}

\section{$\alpha$-Methylacyl CoA racemase (P504S): overview and potential uses in diagnostic pathology as applied to prostate needle biopsies}

\section{A J Evans}

J Clin Pathol 2003;56:892-897

The diagnosis of prostatic adenocarcinoma remains dependent on the recognition of basic haematoxylin and eosin criteria. The discovery of $\alpha$-methylacyl CoA racemase/P504S (AMACR/P504S) overexpression in prostate cancer represents a triumph of high throughput microarray technology, and is a powerful demonstration of how this methodology can be used to facilitate the rapid development of diagnostically relevant antibodies. Immunohistochemistry with anti-AMACR/P504S is useful for detecting prostate cancer in the full range of prostate specimens encountered in surgical pathology, be they needle biopsies, transurethral resection of prostate chips, or prostatectomies. In particular, studies to date with AMACR/P504S clearly demonstrate the ability of this marker to support a diagnosis of malignancy in prostate needle biopsies. This is particularly true when it is combined with negative staining for a basal cell marker, such as $34 \beta E 12$ or p63. Although it has limitations with respect to sensitivity and specificity, AMACR/P504S will no doubt become a standard adjunctive stain used by pathologists seeking to reach a definitive diagnosis in prostate biopsies considered to be atypical, but not diagnostic of malignancy on haematoxylin and eosin sections alone.

Correspondence to: Dr A J Evans, Department of Laboratory Medicine and Pathobiology, University of Toronto, University Health Network, Room 4-302, Princess Margaret Hospital, 610 University Avenue, Toronto, Ontario, Canada, M5G 2M9;

andrew.evans@uhn.on.ca
$\mathrm{T}$ he use of serum prostate specific antigen (PSA) screening to facilitate early detection of prostate cancer has resulted in a dramatic increase in the number of prostate needle core biopsies for pathologists to examine. This has been accompanied by a notable increase in the number of biopsies with microfoci of adenocarcinoma, defined as a single focus of tumour less than $1 \mathrm{~mm}$ in greatest dimension. These microfocal or "minimal" cancers can present a great challenge for pathologists who must try, with confidence, to distinguish them from benign prostate glands. The accepted major haematoxylin and eosin (H\&E) criteria for the diagnosis of prostatic adenocarcinoma include the following: (1) acini that infiltrate the prostatic stroma, particularly in between benign glands; (2) nuclear atypia, with or without prominent macronucleoli; and (3) the absence of a basal cell layer. ${ }^{1}$ In most cases, the diagnosis of minimal adenocarcinoma can be made easily using these criteria. However, there are instances in which H\&E features fall short of those needed for an outright diagnosis of malignancy and additional support is required.

\footnotetext{
"The use of negative staining for basal cell specific cytokeratin does carry several caveats" $^{\prime \prime}$
}

Currently, the absence of a basal cell layer in the suspicious acini, demonstrated by negative immunohistochemical staining for high molecular weight cytokeratin, using the antibodies $34 \beta \mathrm{E} 12$ and/or K903, is the most widely used adjunctive tool. To be interpretable, this approach requires the presence of positive staining of the basal cells in benign glands, which serve as an internal control. Other basal cell specific antibodies such as those directed at cytokeratin $5 / 6^{2}$ and $p 63^{3}$ are emerging, although they are not yet in widespread use. However, the use of negative staining for basal cell specific cytokeratin does carry several caveats. Benign mimics of prostate cancer-including atrophy, post-atrophic hyperplasia, and adenosis/atypical adenomatous hyperplasia (AAH) —often have a discontinuous basal cell layer. The same is true for the putative precursor to invasive adenocarcinoma, high grade prostatic intraepithelial neoplasia (HGPIN). Prolonged formalin fixation has been shown to have a negative effect on the detection of basal cell specific keratins, potentially giving rise to false negative staining. ${ }^{4}$ Finally, morphological variants of prostatic adenocarcinoma, notably the ductal type, may show focally positive basal cell staining. ${ }^{5}$ Given these limitations, a sensitive and specific immunohistochemical marker that stains positively in prostatic adenocarcinoma would be of great value to pathologists faced with worrisome small foci on prostate needle biopsies. The recently described marker P504S, or $\alpha$-methylacyl CoA racemase (AMACR), has great promise in this regard and is the focus of this review.

Abbreviations: $\mathrm{AAH}$, atypical adenomatous hyperplasia; AMACR, $\alpha$-methylacyl CoA racemase; HGPIN, high grade prostatic intraepithelial neoplasia; $\mathrm{H} \& \mathrm{E}$, haematoxylin and eosin; PSA, prostate specific antigen; TURP, transurethral resection of prostate 


\section{DISCOVERY OF AMACR/P504S AS A MARKER OF PROSTATE CANCER}

$\mathrm{Xu}$ et al used a combination of cDNA subtraction and high throughput microarray screening to identify differentially expressed genes that were specific to both prostate tissue in general and prostatic adenocarcinoma. ${ }^{6}$ Subtraction analyses of cDNA libraries created from RNA extracted from prostate tumours, normal prostate tissue, and normal pancreatic tissue resulted in the identification of two genes, P503S and P504S, that were overexpressed in prostate tumours and/ or normal prostate tissue. In confirmatory studies using high throughput cDNA microarray analysis, northern blotting, and quantitative real time polymerase chain reaction (TaqMan methodology), $\mathrm{Xu}$ et al found that P503S was expressed in both prostatic adenocarcinoma and benign prostate tissue. ${ }^{6}$ In contrast, P504S was selectively overexpressed in prostatic adenocarcinoma and showed minimal or undetectable expression in benign prostate tissue. Immunohistochemical staining using rabbit monoclonal antibodies generated against P504S showed positive staining in prostate tumours and negative staining in benign prostate tissue. Using bioinformatics and colony hybridisation, the full length $1621 \mathrm{bp}$ sequence for P504S was cloned and found to encode human $\alpha$-methylacyl CoA racemase (Genbank accession number 4204097). Other groups have independently confirmed the AMACR microarray findings of $\mathrm{Xu}$ et al. ${ }^{78}$ Most recently, Luo et al used quantitative reverse transcription polymerase chain reaction to demonstrate a ninefold upregulation of AMACR mRNA in clinical prostate cancer samples compared with normal prostate tissue. ${ }^{9}$

\section{PHYSIOLOGICAL FUNCTION OF AMACR/P504S AND ITS POTENTIAL ROLE IN THE PATHOGENESIS OF PROSTATE CANCER}

The gene for AMACR is located on chromosome 5pl3 and encodes a 382 amino acid protein that plays a key role in the $\beta$ oxidation of branched chain fatty acids and the bile acid intermediates dihydroxycholestanoic acid and trihydroxycholestanoic acid. Specifically, it catalyses the conversion of $(2 R) \alpha$-methyl branched chain fatty acyl CoAs to their $(S)$ stereoisomers. Only stereoisomers with the 2-methyl group in the $(S)$ position can be metabolised by peroxisomal oxidases, the first enzymes in the $\beta$ oxidation pathway. ${ }^{10-12}$ Subcellular fractionation studies in human liver have demonstrated that AMACR is located in peroxisomes and mitochondria. ${ }^{12}$

Mutations in the AMACR gene, associated with reduced enzyme activity, have been implicated in the development of adult onset sensory motor neuropathy. This is thought to be a consequence of sustained rises in plasma branched chain fatty acids, such as pristinic acid. ${ }^{13}$ With respect to prostate cancer, Zheng and colleagues $^{14}$ found that particular sequence variants in the AMACR gene strongly co-segregate with prostate cancer development in families affected by hereditary prostate cancer. It is also known that men who consume diets rich in dairy products and red meat are at increased risk for developing prostate cancer. ${ }^{15}{ }^{16}$ Dairy products and red meat represent the major dietary sources of the branched chain fatty acid substrates of AMACR/P504S. Thus, there is presumptive evidence that the overexpression of AMACR/P504S coupled with dietary factors could play an important role in prostate cancer progression. The mechanism by which this might occur is a matter of speculation at the present time.

\section{AMACR/P504S EXPRESSION IN NORMAL TISSUES AND MALIGNANCIES OTHER THAN PROSTATE CANCER}

AMACR/P504S overexpression is not unique to prostate cancer. As such, this marker cannot be used to identify prostate cancer in biopsies containing metastatic adenocarcinoma of unknown primary site. In silico analyses of expressed sequence tags and serial analysis of gene expression databases reveal that AMACR is expressed in normal tissue from the nervous system, colon, kidney, breast, and pancreas, in addition to blood elements. ${ }^{17}$ In silico methods also show that cancers arising in the bone marrow, breast, colon, genitourinary system (other than prostate cancer), lung, lymph nodes, nervous system, pancreas, soft tissue, and uterus all show a greater abundance of AMACR/P504S transcripts than do the normal tissues from which they arise. Immunohistochemically, moderate to strongly positive staining with AMACR/P504S has been demonstrated in a wide variety of normal human tissues, including hepatocytes, the epithelium of proximal renal tubules and glomeruli, and the acinar and ductal cells of major salivary glands. ${ }^{9}$ Strongly positive immmunohistochemical staining for AMACR/P504S has been demonstrated in carcinomas of colorectal, ovarian, breast, urothelial, and lung origin, in addition to melanoma and lymphoma. ${ }^{17}$

\section{AMACR/P504S IMMUNOHISTOCHEMICAL STAINING IN PROSTATE TISSUE INCLUDING NEEDLE CORE BIOPSIES}

Several groups have assessed the expression of the AMACR/ $\mathrm{P} 504 \mathrm{~S}$ protein in prostate cancer and benign prostate tissue by immunohistochemistry performed on needle biopsies, ${ }^{8}{ }^{18-20}$ radical prostatectomies, ${ }^{8} 92021$ and tissue microarrays prepared from radical prostatectomies ${ }^{81722}$ and from transurethral resection of prostate (TURP) specimens. ${ }^{18} 20$ The intensity of staining has been assessed predominantly by semiquantitative visual analysis; however, digital image analysis methods have also been used. ${ }^{8}{ }^{17}$ Collectively, these studies have all shown strongly positive AMACR/P504S immunoreactivity in prostatic adenocarcinoma. The staining is uniformly described as being easily visible on low power examination (less than $\times 100$ ), in addition to being circumferential, granular, luminal (apical) to subluminal, or diffusely cytoplasmic in nature. These features constitute a consensus definition of positive AMACR/P504S staining in prostate cancer and are summarised in table 1 . This is in contrast to the focal, weak, non-circumferential positivity seen in the occasional benign gland, and generally absent staining of the prostatic stroma. Figure 1 shows the typical staining patterns for prostate cancer and benign prostate tissue.

\section{"There is presumptive evidence that the overexpression of AMACR/P504S coupled with dietary factors could play an important role in prostate cancer progression"}

At the time of writing, Zeta Corporation produces the only commercially available anti-AMACR/P504S antibody, a rabbit monoclonal. This will pave the way for its widespread use with prostate needle biopsies. With respect to clinical

Table 1 Criteria for immunohistochemical staining with anti- $\alpha$-methylacyl CoA racemase (AMACR/P504S) that would support a diagnosis of malignancy on prostate needle biopsies

Intense staining easily visible on low power examination Circumferential, granular, luminal (apical) to diffuse cytoplasmic staining of acini with malignant features on haematoxylin and eosin sections Negative staining or weak, non-circumferential staining in adjacent benign glands 

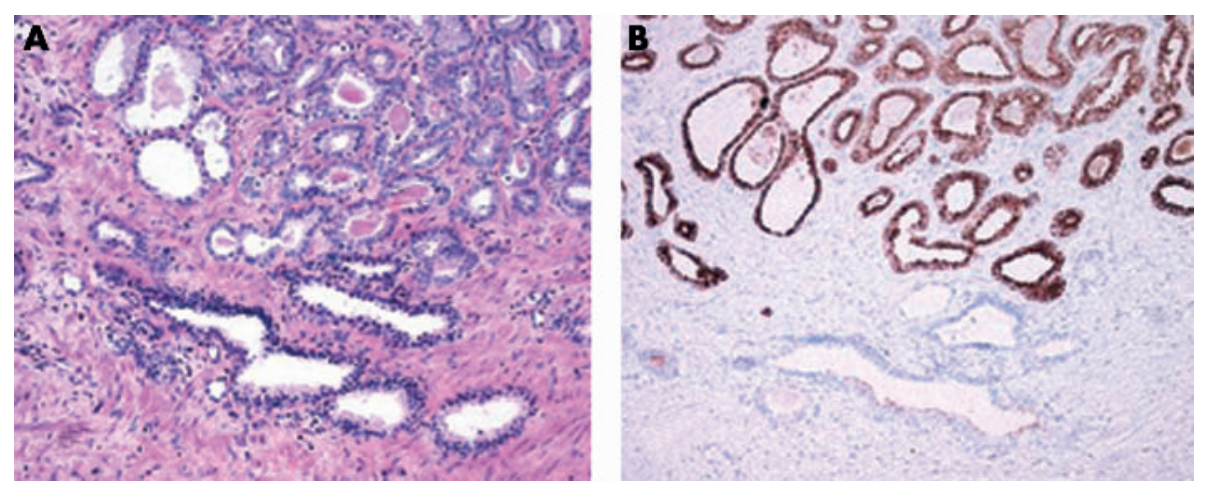

Figure 1 Typical pattern of immunohistochemical staining of prostatic adenocarcinoma and benign prostate glands with antibody directed against $\alpha$-methylacyl CoA racemase (AMACR/P504S). (A) Haematoxylin and eosin stain. (B) Section stained with the rabbit monoclonal anti-AMACR/P504S (Zeta Corporation, Sierra Madre, California, USA). Staining was performed using a 32 minute incubation with the primary antibody (1/100 dilution) and mild $\mathrm{CCl}$ antigen retrieval as part of the Ventana automated immunostaining protocol. Note the intense, circumferential, granular staining in the adenocarcinoma compared with the absence of staining in the adjacent benign glands and stroma (original magnification, $\times 100$ ).

usefulness, the key question is whether or not positive AMACR/P504S staining of small foci of atypical glands can be used to support a diagnosis of cancer. Four published studies (table 2) have specifically dealt with this sensitivity and specificity question in needle biopsies. Overall, they show sensitivities that range from $82 \%$ to $100 \%$ and specificities ranging from $79 \%$ to $100 \%$. With respect to the detection of microfocal or minimal adenocarcinoma on needle biopsies, three of the studies in table 2 provide data on this question. Jiang and colleagues ${ }^{21}$ initially looked at seven biopsies and have subsequently reported their experience on a larger series of 73 biopsies, ${ }^{18}$ with foci of adenocarcinoma, $<1 \mathrm{~mm}$ in maximum dimension, for which the malignant acini showed an absence of basal cell staining for $34 \beta E 12$. All examples were positive for AMACR/P540S, with negative staining in benign glands. In all cases, the malignant acini were positive for AMACR/P504S, with negative staining in benign glands. The study of Rubin et al included a subset of 26 "diagnostically challenging" needle biopsies for which the final diagnosis of adenocarcinoma required expert review in conjunction with a basal cell specific stain (34ßE12 or p63). ${ }^{8}$ Many of these 26 cases consisted of four to five glands, and would have had a maximum dimension of less than $1 \mathrm{~mm}$. For these diagnostically challenging foci, the sensitivity of AMACR staining ranged from $94.5 \%{ }^{18}$ to $100 \%{ }^{21}$ Iczkowski et al looked at the ability of AMACR/P504S immunostaining to help reach a definitive diagnosis in a series of 43 needle biopsies considered to be "atypical small acinar proliferations suspicious for, but not diagnostic of malignancy", based on H\&E criteria. ${ }^{23}$ Presented in abstract form, these investigators reported that the use of AMACR/ $\mathrm{P} 504 \mathrm{~S}$ in conjunction with immunostaining for $34 \beta \mathrm{E} 12$ enabled them to reach a definitive diagnosis of malignancy or benignancy in $80 \%$ of these cases. Figures 2 and 3 demonstrate the typical and highly effective manner in which immunohistochemical staining with AMACR/P504S can be used to highlight small foci of adenocarcinoma in needle biopsies.

\begin{abstract}
"Knowledge of the frequency with which high grade prostatic intraepithelial neoplasia and other entities that may mimic prostate cancer in prostatic needle biopsies shows positive AMACR/P504S immunoreactivity is crucial to the interpretation of this marker"
\end{abstract}

The studies summarised above clearly show that not all prostate cancers will be positive for AMACR/P504S and not all benign tissue will be negative. A major factor contributing to this variability could be the different anti-AMACR/P504S antibodies used in these studies. To underscore this point, Kunju and colleagues ${ }^{24}$ compared a polyclonal anti-AMACR to the Zeta Corporation monoclonal anti-P504S antibody using tissue microarrays prepared from prostatectomy specimens. The polyclonal anti-AMACR was $100 \%$ sensitive and specific for prostate cancer, whereas the monoclonal antiP504S was $94 \%$ sensitive and 70\% specific. Other contributing factors to variable sensitivity and specificity of AMACR/P504S staining include: different concentrations of the primary antibodies, manual versus automated staining techniques, and different protocols for antigen retrieval. The impact of the duration of formalin fixation on AMACR/P504S staining has not been evaluated and may be of importance, as has been shown to be the case for the immunohistochemical detection of prostatic basal cells with $34 \beta \mathrm{E} 12 .^{4}$

Table $2 \alpha$-Methylacyl CoA racemase (AMACR/P504S) immunohistochemical staining of prostate needle biopsies: summary of published data

\begin{tabular}{|c|c|c|c|c|}
\hline First author & Antibody used & $\begin{array}{l}\text { Number of biopsies } \\
\text { (malignant/benign) }\end{array}$ & $\begin{array}{l}\text { Malignant foci with } \\
\text { positive staining }\end{array}$ & $\begin{array}{l}\text { Benign foci with positive } \\
\text { staining* }\end{array}$ \\
\hline Jiang $^{21}$ & $\begin{array}{l}\text { Rabbit monoclonal anti-P504S (Abgenix Biopharma, } \\
\text { Vancouver, Canada) }\end{array}$ & $112(58 / 54)$ & $58 / 58+$ & $8 / 54$ \\
\hline Rubin $^{8}$ & Polyclonal anti-AMACR (gift from Dr R Wanders) & $94(70 / 24)$ & 68/70‡ & $0 / 24$ \\
\hline Jiang $^{18}$ & Rabbit monoclonal anti-P504S & $142(73 \S / 69)$ & $69 / 73(95 \%)$ & $0 / 69(0 \%)$ \\
\hline Beach $^{19}$ & Rabbit monoclonal anti-P504S (clone 13H4) & $376(186,190)$ & $153 / 186(82 \%)$ & $81 / 377(21 \%)$ \\
\hline
\end{tabular}

*Includes normal, hyperplastic, and atrophic glandular epithelium and excludes high grade prostatic intraepithelial neoplasia; $† 7$ biopsies with foci of adenocarcinoma $<1 \mathrm{~mm}$ in maximum dimension; all 7 showed positive AMACR/P504S staining; $¥ 26 / 70$ biopsies had foci of adenocarcinoma requiring $34 \beta \mathrm{E} 12$ to support a malignant diagnosis; §all 73 biopsies had foci of adenocarcinoma $<1 \mathrm{~mm}$ in maximum dimension supported by confirmatory $34 \beta \mathrm{E} 12$ staining. 

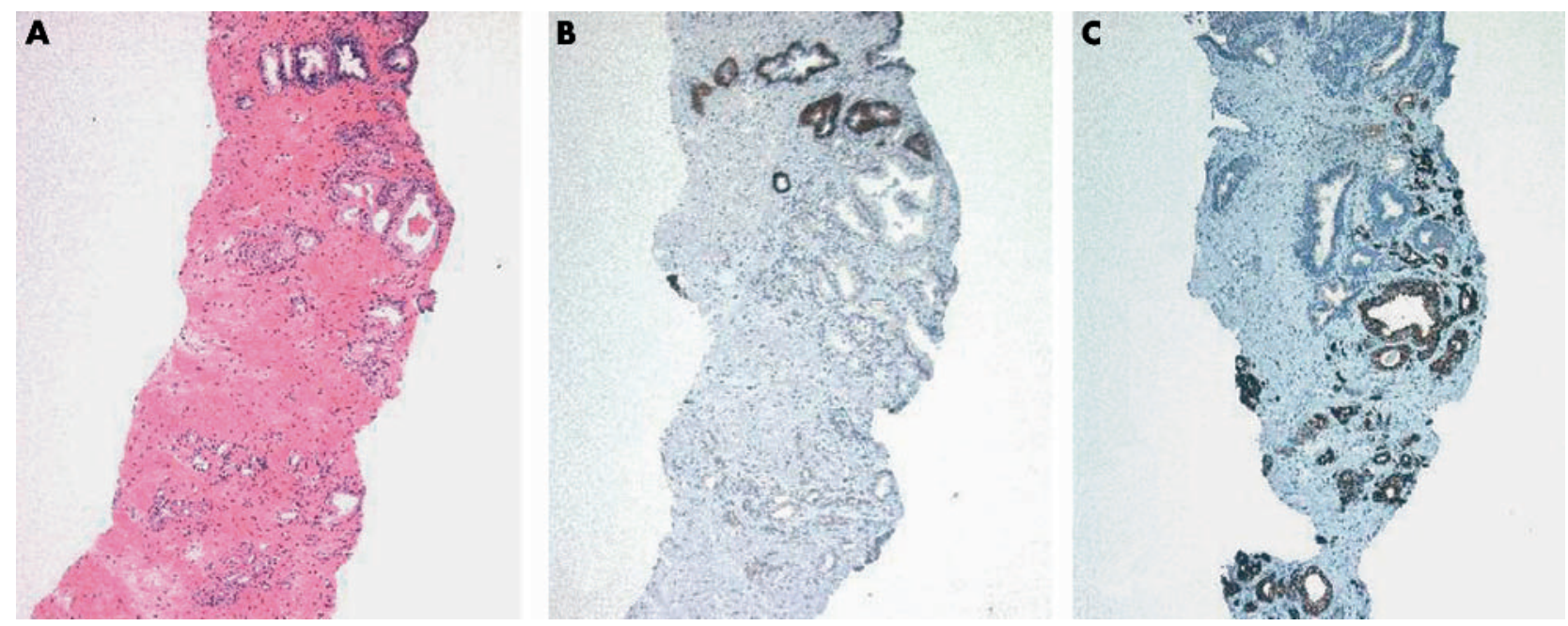

Figure 2 Small focus of prostatic adenocarcinoma on a needle biopsy. (A) Haematoxylin and eosin. (B) Immunostaining for 34ßE12; note the absence of staining for basal cells within the malignant glands as compared with the adjacent benign glands. (C) Malignant glands showing intensely positive, circumferential staining for AMACR/P504S compared with negative staining in the benign glands. A-C: original magnification, $\times 50$.

\section{AMACR/P504S STAINING IN HGPIN AND BENIGN MIMICS OF PROSTATE CANCER}

Knowledge of the frequency with which HGPIN and other entities that may mimic prostate cancer in prostatic needle biopsies shows positive AMACR/P504S immunoreactivity is crucial to the interpretation of this marker. Moderate to strongly positive staining has been reported for HGPIN in more than $64 \%$ of foci. ${ }^{17}$ Jiang et al also reported strongly
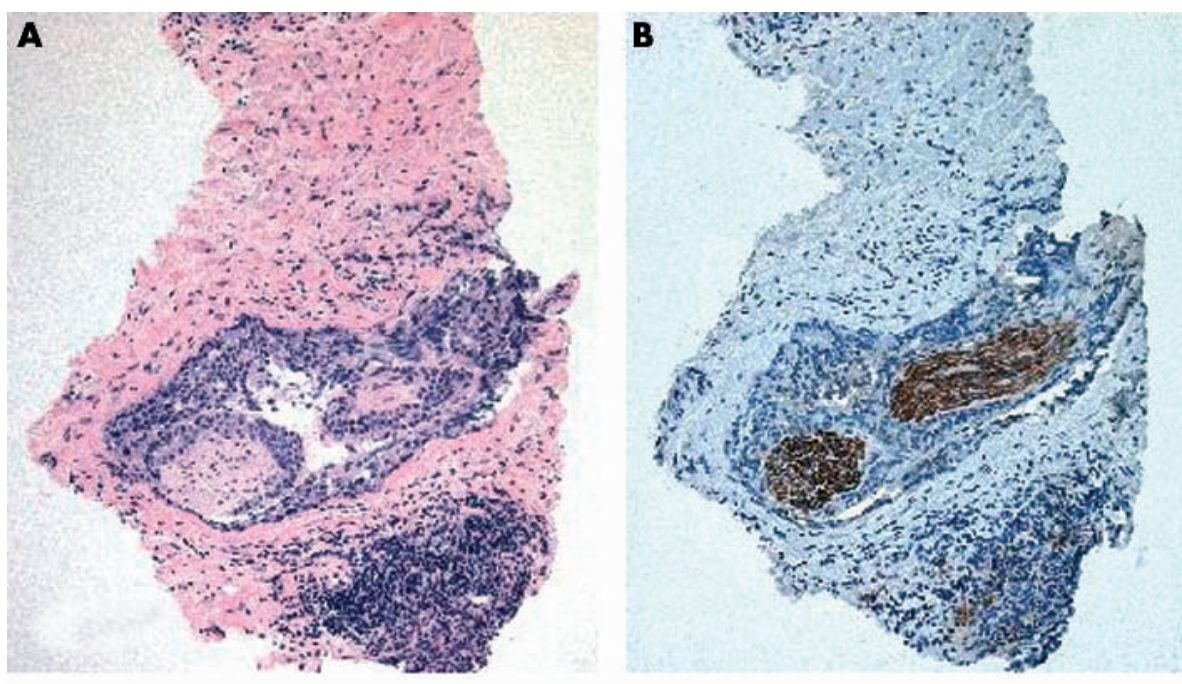

Figure 3 Another example of a small focus of prostatic adenocarcinoma on a needle biopsy. (A) Haematoxylin and eosin stained section showing adenocarcinoma with perineural invasion. (B) S-100 staining to highlight the presence of the involved nerve. (C) $34 \beta E 12$ staining to show the absence of a basal cell layer. (D) $\alpha$-Methylacyl CoA racemase (AMACR/P504S) staining that is restricted to the adenocarcinoma. A-D: original magnification, $\times 50$.
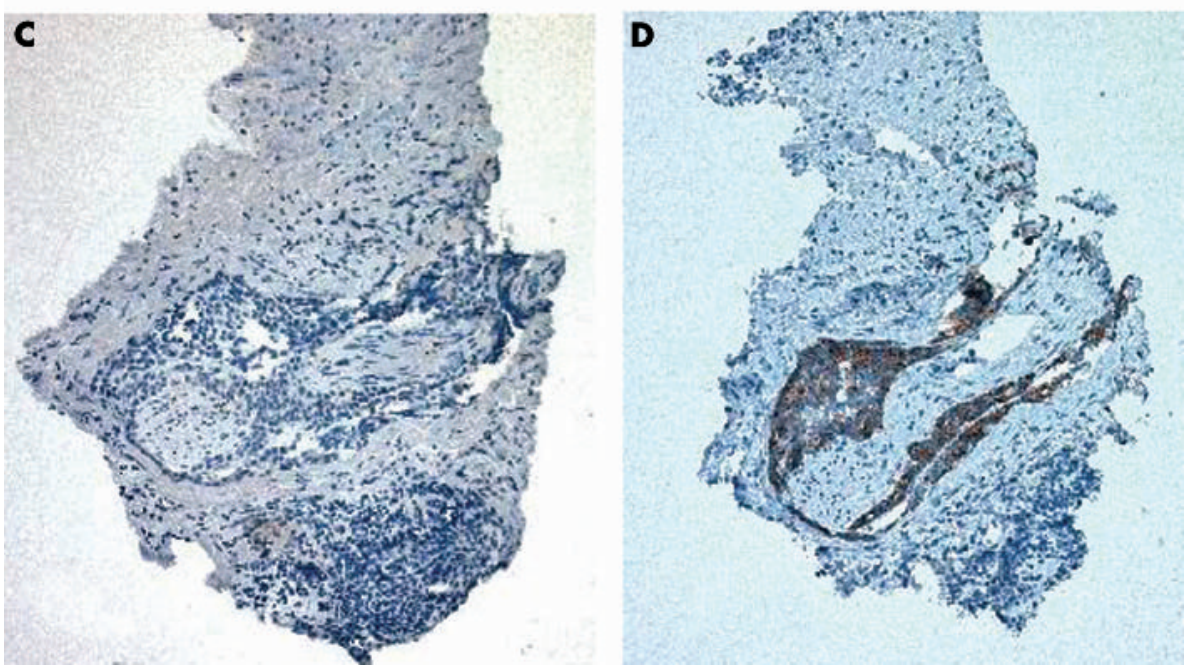
positive staining in HGPIN, and noted that when HGPIN partially involved a gland, staining was confined to the HGPIN and did not extend into the normal epithelial cells within the same gland. ${ }^{21}$ With respect to staining intensities, Luo et al reported that both invasive carcinoma and HGPIN had higher immunohistochemical staining scores than normal prostate epithelium; ; however, the score for carcinoma was significantly higher than that for HGPIN. Similar results were also reported by Rubin et al. ${ }^{8}$

The widely recognised "pseudoneoplasms" that can mimic prostatic adenocarcinoma on needle biopsies include atypical adenomatous hyperplasia (AAH) or adenosis, atrophy, postatrophic hyperplasia, basal cell metaplasia, and seminal vesicle or ejaculatory duct epithelium. The frequency of positive AMACR/P504S staining in foci of AAH was specifically assessed by Yang et al in 40 examples identified in prostatectomies, needle biopsies, and TURP specimens. ${ }^{20}$ Basal cell specific staining for $34 \beta \mathrm{E} 12$ confirmed the presence of patchy basal cells in all 40 cases. In 33 of 40 examples of AAH there was no detectable staining for AMACR/P504S, although there was focal staining in four of 40 and diffusely positive staining in three of 40 . Prostatic carcinomas and benign prostatic hyperplasia served as positive and negative controls and showed the predicted staining patterns. As such, these authors concluded that AMACR/P504S immunostaining distinguishes most, but not all, cases of AAH from adenocarcinoma. Weak, positive staining in AAH has also been reported by Jiang et al in three of 11 cases. ${ }^{21}$

"AMACR/P504S immunostaining distinguishes most, but not all, cases of atypical adenomatous hyperplasia from adenocarcinoma"

Foci of atrophic prostate glands have been noted to show moderate, focal, non-circumferential positive AMACR/P504S staining in $0 \%$ to $36 \%{ }^{17}{ }^{19}{ }^{21}$ of the examples published to date. In addition, Rubin and colleagues ${ }^{8}$ used quantitative image analysis and found that the staining intensity in atrophic foci was greater than that of non-atrophic benign prostate epithelium, but less than that for prostate cancer. The study by Beach et al is the only one that reported the AMACR/P504S staining frequency in post-atrophic hyperplasia. ${ }^{19}$ Staining was found to be negative in all 10 examples found in their series of 376 prostate needle biopsies. Beach et al also reported an absence of staining in seminal vesicles, basal cell hyperplasia, and transitional cell metaplasia. ${ }^{19}$ In addition, these investigators noted that adjacent inflammation appeared to have no effect on the presence or absence of staining for AMACR/P504S in benign glands.

\section{AMACR/P504S STAINING IN RELATION TO HISTOPROGNOSTIC FACTORS IN PROSTATE CANCER}

The data that have been generated to date indicate that there is no association between AMACR/P504S immunoreactivity and histoprognostic factors, such as Gleason score, pathological stage, or margin status. ${ }^{8} 9$ In addition, no association has been noted between AMACR/P504S protein expression and preoperative serum PSA values, the incidence of postoperative PSA failures in a period of up to three years, and the Ki67 labelling index as a marker of tumour cell proliferation. ${ }^{8}$

Strong positive AMACR/P504S immunoreactivity is also present in unusual morphological variants of prostate cancer. Beach et al reported positive staining in five of six cancers with pseudohyperplastic patterns, two of two with atrophic patterns, two of three with mucinous morphology, and five of five examples of ductal adenocarcinoma. ${ }^{19}$

\section{Take home messages}

- Positive staining for $\alpha$-methylacyl CoA racemase (AMACR/P504S) can be used to support a diagnosis of cancer on prostate needle core biopsies when the focus in question is $<1 \mathrm{~mm}$ in maximum dimension

- AMACR/P504S staining must be interpreted in the context of basic haematoxylin and eosin criteria for malignancy, plus/minus other supportive studies, such as a basal cell specific markers like $34 \beta E 12$ or p63

- High grade prostatic intraepithelial neoplasia and other benign mimics of prostate cancer show variable positive immunoreactivity for AMACR/P504S

\section{AMACR/P504S STAINING IN PROSTATE CANCER TREATED WITH RADIATION AND ANTI-ANDROGEN TREATMENT}

Current data show that the treatment of prostate cancer with either of these modalities has no overall effect on AMACR/ P504S overexpression by the tumour, demonstrating that this immunostain is useful in the detection of cancer in posttreatment biopsies. Jiang and colleagues ${ }^{25}$ found that radiation treatment had no impact on AMACR/P504S staining in needle biopsies, TURPs, or salvage radical prostatectomies. In addition, there was no correlation with the intensity of staining and the degree of treatment effect. Benign glands also showing radiation atypia were found to stain negatively.

When comparing clinically localised prostate cancer with metastatic, hormone refractory tumours, Kuefer and colleagues $^{22}$ and Rubin and colleagues ${ }^{8}$ reported a significant decrease in AMACR/P504S protein expression in hormone refractory tumours. Beach et al also reported a decrease in staining intensity in prostate cancer treated with antiandrogens, which was proportional to the degree of treatment effect seen. ${ }^{26}$ Interestingly, these observations do not seem to be directly attributable to the anti-androgen treatment itself based on studies performed on prostate cancer cell lines. Such experiments have provided evidence that AMACR/P504S expression is not hormone dependent. ${ }^{22}$

\section{FUTURE DIRECTIONS}

In the future, we will probably see the use of combined AMACR/P504S basal cell marker "immunococktails", in addition to those already published for the basal cell markers $34 \beta \mathrm{E} 12$ and p63 alone. ${ }^{27}$ We will also see the development of other immunomarkers thought to be selectively overexpressed in prostate cancer, such as hepsin and $\mathrm{PIMl}^{28}$ in addition to the PrLz protein. ${ }^{29}$ All of these approaches will enhance the detection of prostate cancer in needle biopsies. They should also result in a large decrease in the number of suspicious, but non-diagnostic, biopsies and the number of repeat biopsies that would follow.

\section{REFERENCES}

1 Thorson P, Humphrey PA. Minimal adenocarcinoma in prostate needle biopsy tissue. Am J Clin Pathol 2000;114:896-909.

2 Abrahams N, Ormsby A, Brainard J. Cytokeratin 5/6 is a basal cell-specific marker in benign prostatic glandular proliferations and in prostate tissue with androgen deprivation effect. Mod Pathol 2002:15:150A

3 Weinstein MH, Signoretti S, Loda M. Diagnostic utility of immunohistochemical staining for $\mathrm{p} 63$, a sensitive marker of prostatic basal cells. Mod Pathol 2002:15:1302-8.

4 Varma $M$, Linden MD, Amin MB. Effect of formalin fixation and epitope retrieval techniques on antibody 34 betaE 12 immunostaining of prostatic tissues. Mod Pathol 1999;12:472-8.

5 Oliai BR, Kahane H, Epstein Jl. High molecular weight cytokeratin (HMWCK) reactivity in prostate carcinoma. Mod Pathol 2002;15:176A. 
6 Xu J, Stolk JA, Zhang X, et al. Identification of differentially expressed genes in human prostate cancer using subtraction and microarray. Cancer Res 2000;60:1677-82.

7 Welsh JB, Sapinoso LM, Su Al, et al. Analysis of gene expression identifies candidate markers and pharmacological targets in prostate cancer. Cancer Res 2001;61:5974-8.

8 Rubin MA, Zhou M, Dhanasekaran SM, et al. Alpha-methylacyl coenzyme A racemase as a tissue biomarker for prostate cancer. JAMA 2002;287:1662-70.

9 Luo J, Zha S, Gage WR, et al. Alpha-methylacyl-CoA racemase: a new molecular marker for prostate cancer. Cancer Res 2002;62:2220-6.

10 Schmitz W, Albers C, Fingerhut R, et al. Purification and characterization of an alpha-methylacyl-CoA racemase from human liver. Eur $J$ Biochem 1995;231:815-22.

11 Van Veldhoven PP, Croes K, Casteels M, et al. 2-methylacyl racemase: a coupled assay based on the use of pristanoyl-CoA oxidase/peroxidase and reinvestigation of its subcellular distribution in rat and human liver. Biochim Biophys Acta 1997;1347:62-8.

12 Ferdinandusse S, Denis S, IJlst L, et al. Subcellular localization and physiological role of alpha-methylacyl-CoA racemase. J Lipid Res 2000;41:1890-6.

13 Ferdinandusse S, Denis S, Clayton PT, et al. Mutations in the gene encoding peroxisomal alpha-methylacyl-CoA racemase cause adult-onset sensory motor neuropathy. Nat Genet 2000;24:188-91.

14 Zheng SL, Chang BL, Faith DA, et al. Sequence variants of alpha-methylacyl$\mathrm{CoA}$ racemase are associated with prostate cancer risk. Cancer Res 2002;62:6485-8.

15 Chan JM, Giovannucci EL. Dairy products, calcium, and vitamin D and risk of prostate cancer. Epidemiol Rev 2001;23:87-92.

16 Kolonel LN. Fat, meat, and prostate cancer. Epidemiol Rev 2001;23:72-81.

17 Zhou M, Chinnaiyan AM, Kleer CG, et al. Alpha-methylacyl-CoA racemase: a novel tumor marker over-expressed in several human cancers and their precursor lesions. Am J Surg Pathol 2002;26:926-31.
18 Jiang Z, Wu CL, Woda BA, et al. P504S/alpha-methylacyl-CoA racemase: a useful marker for diagnosis of small foci of prostatic carcinoma on needle biopsy. Am J Surg Pathol 2002;26:1169-74.

19 Beach R, Gown AM, Peralta-Venturina MN, et al. P504S immunohistochemical detection in 405 prostatic specimens including 376 18gauge needle biopsies. Am J Surg Pathol 2002;26:1588-96.

20 Yang XJ, Wu CL, Woda BA, et al. Expression of alpha-methylacyl-CoA racemase (P504S) in atypical adenomatous hyperplasia of the prostate. Am J Surg Pathol 2002;26:921-5.

21 Jiang Z, Woda BA, Rock KL, et al. P504S: a new molecular marker for the detection of prostate carcinoma. Am J Surg Pathol 2001;25:1397-404.

22 Kuefer R, Varambally S, Zhou M, et al. Alpha-methylacyl-CoA racemase: expression levels of this novel cancer biomarker depend on tumor differentiation. Am J Pathol 2002;161:841-8.

23 Iczkowski KA, Jiang Z, Tretiakova M, et al. Prostatic needle biopsies with suspicious (ASAP) diagnosis: $80 \%$ resolved using P504S/AMACR and keratin 34bE12 immunostaining. Mod Pathol 2003;16:154A.

24 Kunju LP, Rubin MA, Shen R, et al. Comparison of monoclonal antibody (P504S) and polyclonal antibody to alpha-methylacyl-CoA racemase in benign atypical and malignant prostate tissues. Mod Pathol 2003;16:158A.

25 Jiang Z, Tretiakova M, Steinberg G, et al. Overexpression of alphamethylacyl-CoA racemase/P504S in recurrent prostatic adenocarcinoma after radiation. Mod Pathol 2003;16:155A.

26 Amin M, Beach R, Gown AM, et al. Use of a novel immunohistochemical (IHC) panel (P504S, p63 and 34BE12) in the diagnosis of post-radiation therapy (PRT) prostate cancer (PCa). Mod Pathol 2003;16:139A.

27 Zhou M, Shah R, Shen R, et al. Basal cell cocktail (34betaE12+p63) improves the detection of prostate basal cells. Am J Surg Pathol 2003:27:365-71.

28 Dhanasekaran SM, Barrette TR, Ghosh D, et al. Delineation of prognostic biomarkers in prostate cancer. Nature 2001;412:822-6.

29 Harik L, Cui LY, Sen B, et al. Immunohistochemical analysis of a novel prostate epithelial cell-specific gene "PrLZ" protein in prostatic epithelia in radical prostatectomy specimens. Mod Pathol 2003;16:153A. 